Chirurg 2011 · 82:389-390

DOI 10.1007/s00104-010-2002-7

Online publiziert: 29. April 2011

(c) Springer-Verlag 2011

\author{
C.T. Germer \\ Klinik und Poliklinik für Allgemein-, Viszeral-, Gefäß- und Kinderchirurgie, \\ Universitätsklinikum Würzburg
}

\title{
Single-Inzision in der minimal-invasiven Chirurgie
}

rurgie führt nicht logischerweise in eine technisch einfach durchzuführende und praktikable Operationstechnik. Vielmehr zeigte die weitere Entwicklung, dass nicht die Machbarkeit eines Verfahrens die Antriebskraft für die Weiterentwicklung ist, sondern die dahinter stehende Idee und Inspiration. So überzeugten die Ergebnisse der sog. „Hybrid-NOTES-Techniken“ durch ihre technische und komplikationsarme Machbarkeit.

Die „single access surgery“, Minilaparoskopie und „reduced port surgery“ entstanden sicherlich nicht zuletzt als Resultat einer weiteren (praktikablen) laparokopischen Minimalisierung. Jedoch handelt es sich bei diesen Techniken auch nicht um chirurgisches Neuland. So führte bereits in den 7oer Jahren des 20. Jahrhunderts Reimund Wittmoser [2] in Düsseldorf thorakoskopische Single-PortEingriffe mit einem Multikanaloperationsendoskop durch. 1985 erfolgte die erste laparoskopische Cholezystektomie durch Erich Mühe [3] aus Böblingen, ebenfalls in Single-Port-Technik mit einem als "Galloskop“ bezeichneten Multikanaloperationstrokar. Schließlich ist als Vertreter der Single-Access-laparoskopischen Cholezystektomie der italienische Chirurg Giuseppe Navarra [4] zu nennen, der 1997 über eine singuläre Hautinzision und hierüber eingebrachte Trokare die „One-wound-Cholezystektomy“ erfolgreich durchführte. Die genaue Betrachtung dieser Methoden impliziert bereits eine Begriffsdefinition innerhalb der "single incision surgery“. Die beiden erstgenannten Techniken beinhalten eine singuläre Haut- und Faszieninzision und die Verwendung eines Multikanalportsystems, über welches die Optik und al- le notwendigen Instrumente geführt werden. Die von Navarra publizierte Methode beschreibt eine singuläre Hautinzision mit anschließendem Platzieren von mehreren Arbeitstrokaren für die Instrumentenführung. Möglicherweise spiegelt sich diese nicht von Anbeginn an klare Begriffsdefinition und Trennschärfe der einzelnen Techniken in der Vielzahl der zum Teil sehr wohlklingenden Namensgebungen für diese Methode wieder.

Im Unterschied zur Etablierung der konventionell laparoskopischen Chirurgie führte die Einführung der Single-AccessMethode nicht etwa zu einer Erweiterung des bestehenden operativen Spektrums, sondern vielmehr zu einer technisch ergonomischen Veränderung und Herausforderung in der Durchführung der bereits sehr gut etablierten konventionell laparoskopischen Eingriffe. Wie am Beispiel der praktischen Umsetzung reiner NOTESVerfahrens gezeigt, führte auch in der laparoskopischen Single-Access-Chirurgie die alleinige Verbindung von hervorragenden laparoskopischen Fähigkeiten mit entsprechend konzipierten technischen Tools nicht zu einer umgehenden und unproblematischen Umsetzung der Single-Port-Verfahren im chirurgisch-praktischen Alltag.

\section{Wie viel Minimalisierung ist sinnvoll?}

Die Fähigkeit zur Durchführung von Single-Port/Access-Eingriffen bedarf aufgrund verschiedener, insbesondere jedoch technischer Anforderungen durchaus einer systematisierten Ausbildung in dieser Operationstechnik. Es ist deshalb nicht verwunderlich, sondern überaus scher Möglichkeiten mit entsprechend hochqualifizierter laparoskopischer Chi- 
sinnvoll, dass Paul Curcillo für die stufenweise Reduktion der Trokarinzisionen und das Konzept der „reduced port surgery“ plädiert. Obwohl in der Zwischenzeit auch anspruchsvolle laparoskopische Operationen im Single-Access-Verfahren durchgeführt werden, sollten diese spezialisierten, laparoskopisch erfahrenen Chirurgen vorbehalten sein. Insbesondere vor dem Hintergrund, dass im Moment noch keine zuverlässige Aussage hinsichtlich des tatsächlichen Benefits für die Patienten getroffen werden kann. Es stehen weiterhin die Fragen im Raum, wie viel Minimalisierung der minimal-invasiven Techniken wissenschaftlich nachweisbar sinnvoll ist und ob die alleinige Minimalisierung der Inzisionen und Anwendung von sog. Miniinstrumenten bereits zu vergleichbaren Ergebnissen wie in Hybrid-NOTES- und Single-Port/AccessVerfahren führen.

Die Anforderung an den laparoskopischen Chirurgen von heute lautet, mehr denn je gleichsam aufgeschlossen und kritisch allen Verfahren gegenüber zu stehen. Die laparoskopische Chirurgie wird wie nie zuvor von einer Methodenvielfalt geprägt. Es ist die Aufgabe der Chirurgen, diese neuen Wege ärztlich und wissenschaftlich ehrlich zu erkunden. Erst die genaue Auseinandersetzung und kritische Evaluation aller bestehenden $\mathrm{Me}$ thoden erlaubt deren effektiven und differenzierten Einsatz und lässt Raum für die ukunftsvision einer sicheren und auf jeden Patienten individuell zugeschnittenen minimal-invasiven Chirurgie nach dem Konzept eines „tailored approach“.

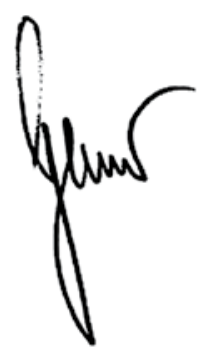

Prof. Dr. C.T. Germer

\section{Korrespondenzadresse}

\section{Prof. Dr. C.T. Germer}

Klinik und Poliklinik für Allgemein-, Viszeral-, Gefäß- und Kinderchirurgie, Universitätsklinikum Würzburg, Oberdürrbacher Str. 6, 97080 Würzburg

Germer_c@klinik.uni-wuerzburg.de

\section{Literatur}

1. Marescaux J, Dallemagne B, Perretta S (2007) Surgery without scars: a report of transluminal cholecystectomy in a human being. Arch Surg 142(9):823-826

2. Wittmoser R (1964) Die Chirurgie der vegetativen Nerven beim Ulcus pepticum. Langenbecks Arch Surg 308(1):387-397

3. Mühe E (1992) Laparoscopic cholecystectomy follow up. Endoscopy 24:754-758

4. Navarra G, Pozza E, Occhionorelli S et al (1997) One-wound laparoscopic cholecystectomy. Br J Surg 84:695

\section{www.SpringerMedizin.de/chirurgie}

\section{Alles rund um Ihr Fachgebiet}

Wählen Sie ab sofort den direkten Weg zu News

und Fachbeiträgen aus Ihrem Fachgebiet:

Auf www.SpringerMedizin.de/chirurgie

halten wir Sie immer auf dem Laufenden.

Hier erwartet Sie ein umfassendes Informationsangebot:

- News aus Berufs- und Gesundheitspolitik

- Ausgewählte Meldungen und Beiträge aus Ihrem Fachgebiet

- Die wichtigsten Studien und Publikationen - für Sie zusammengefasst und kommentiert

- Kompetente Unterstützung bei schwierigen Fragestellungen durch unsere Expertenräte

- Medizinische Fortbildung in Form von Bilderstrecken, Podcasts bis hin zu Reviews und CME-Beiträgen aus den Fachzeitschriften von Springer Medizin

So einfach geht's: Nach einmaliger Registrierung steht Ihnen ein umfangreiches kostenloses Angebot zur Verfügung.

SpringerMedizin.de - Das Internet der Ärzte 